\title{
Clinicopathological features and prognostic factors in angiosarcoma: A retrospective analysis of 200 patients from a single Chinese medical institute
}

\author{
LEI WANG $^{1,2^{*}}$, I WENG LAO $^{1,2^{*}}$, LIN YU $^{1,2}$ and JIAN WANG ${ }^{1,2}$ \\ ${ }^{1}$ Department of Pathology, Fudan University Shanghai Cancer Center, Shanghai 200032; ${ }^{2}$ Department of Oncology, \\ Shanghai Medical College, Fudan University, Shanghai 200433, P.R. China
}

Received December 18, 2015; Accepted May 23, 2017

DOI: $10.3892 / \mathrm{ol} .2017 .6892$

\begin{abstract}
Angiosarcoma is a rare soft tissue sarcoma, and the data about its clinicopathological features and prognostic factors are limited. The purpose of the present study was to report a large series of angiosarcoma at a single institution. Clinical data from 200 cases of angiosarcoma from the Shanghai Cancer Center (Shanghai, China) between March 2006 and March 2014 were retrospectively analyzed. The study population included 97 males and 103 females with ages between 4 and 91 years (median, 53 years). According to the tumor location, 200 cases were divided into 4 groups: i) Tumors involving the head and neck; ii) breast; iii) viscera (including internal organs and bone); and iv) soft tissue (including trunk and extremities). Of the 113 patients with follow-up data, 46 patients succumbed to the disease with a median interval of 10 months. Tumor recurrence/metastasis was identified in 66 patients with a median interval of 4 months. The disease-free survival (DFS) rate at 5-years was $19.3 \%$ and the overall survival (OS) rate at 5-years was $40.8 \%$. Site of tumor origin, size $(\geq 5 \mathrm{~cm})$ and histological differentiation influenced DFS $(\mathrm{P}=0.032,0.038$ and $<0.001$, respectively), and $\mathrm{OS}(\mathrm{P}<0.001,0.008$ and $<0.001$, respectively) rates. Age ( $<65$ years $)$ and multimodal treatment correlated with improved OS $(\mathrm{P}=0.003$ and $<0.001$, respectively). Tumor differentiation and treatment modality were identified to be independent determinants of OS $(\mathrm{P}<0.001$ and 0.038 , respectively). Tumor recurrence/metastasis was an independent predictor of DFS $(\mathrm{P}<0.001)$. The prognosis of angiosarcoma is poor and the mortality rate is high. The
\end{abstract}

Correspondence to: Dr Jian Wang, Department of Pathology, Fudan University Shanghai Cancer Center, 270 Dong An Street, Shanghai 200032, P.R. China

E-mail: softtissuetumor@163.com

${ }^{*}$ Contributed equally

Key words: angiosarcoma, soft tissue sarcoma, clinicopathological features, prognostic factors, retrospective analysis site of tumor origin, size, histological differentiation, age, treatment modality and tumor recurrence/metastasis are all significant prognostic factors. In the present study, multimodal treatment may improve the clinical outcome of patients with angiosarcoma.

\section{Introduction}

Angiosarcoma is a rare type of soft tissue sarcoma with the morphological and immunohistochemical features of endothelial cells. It accounts for $1-4 \%$ of all soft tissue sarcoma cases $(1,2)$. Angiosarcomas are a heterogeneous group of tumors. The primary sites of angiosarcoma include the skin, soft tissue and viscera. Clinical presentation varies considerably depending on the primary site; the majority of cutaneous angiosarcomas present as bruise-like patches, violaceous nodules or plaques, whereas angiosarcomas of soft tissue present as an enlarged painful mass. The clinical presentation of angiosarcoma of the viscera is variable, including abdominal pain, hepatosplenomegaly or anemia (3).

The prognosis for angiosarcoma is also associated with the primary site of the tumor. Fayette et al (3) retrospectively analyzed the clinicopathological features of 161 cases of angiosarcoma, and demonstrated that the patients' survival was significantly impacted by the primary site. A primary site in the liver or heart was associated with a poor prognosis, whereas primary soft tissue tumors were associated with a relatively improved prognosis. Fury et al (4) identified that the progression-free survival time for scalp angiosarcoma was 6.8 months, whereas it was 2.8 months for sites below the clavicle. Due to the rarity of this tumor, the clinicopathological features and prognostic factors require further investigation. At present, there remains a lack of consensus on the treatment strategy for this rare type of tumor. Nevertheless, it is of paramount importance for pathologists to make an accurate diagnosis, as misdiagnosis may lead to inappropriate administration and incorrect assessment of the prognosis.

To better understand the clinicopathological features, treatment response and clinical outcomes of angiosarcoma, an analysis was performed on 200 consecutive cases, the largest series to date, with emphasis on defining the prognostic factors of this rare sarcoma. 


\section{Materials and methods}

Case selection. Between March 2006 and March 2014, 200 consecutive cases of angiosarcoma were identified from the consultation files and surgical pathology files of the Department of Pathology, Fudan University Shanghai Cancer Center, Fudan University (Shanghai, China). The present retrospective study was approved by The Clinical Research Ethics Committee of Fudan University Shanghai Cancer Center. Written informed consent was obtained from the patients or guardians on behalf of the minors involved in the present study. Diagnosis of angiosarcoma was made according to the World Health Organization classification of Tumours of Soft Tissue and Bone (5). Clinical data, including age, sex, tumor location and size, therapy, pattern of recurrence, occurrence of metastasis, and clinical outcome, were obtained from the medical records, pathology reports or discharge summaries. The follow up information was measured via medical records and telephone interviews.

Immunohistochemical staining. All specimens were fixed in $10 \%$ formalin and processed routinely for paraffin embedding. $4 \mu \mathrm{m}$-thick sections were cut and stained with hematoxylin and eosin. The diagnosis of each case was confirmed by two experienced pathologists and representative paraffin blocks containing tumor material were selected for immunohistochemical analysis. The sections were cut and deparaffinized according to standard histological techniques. Endogenous peroxidase activity was blocked by soaking the slides in $0.3 \% \mathrm{H}_{2} \mathrm{O}_{2}$ in methanol for $30 \mathrm{~min}$ at $37^{\circ} \mathrm{C}$. Antigen retrieval was performed by high pressure repair in $0.1 \mathrm{M}$ citrate solution ( $\mathrm{pH}$ 6.0) for $10 \mathrm{~min}$, followed by incubation with the primary antibodies overnight at $4{ }^{\circ} \mathrm{C}$. The primary antibodies used in the study included cluster of differentiation (CD)31 (dilution 1:100; cat. no. JC70A; Dako; Agilent Technologies, Inc., Santa Clara, CA, USA), CD34 (dilution 1:100; cat. no. QBEnd 10; Dako; Agilent Technologies, Inc.), Friend leukemia virus integration 1 (FLI1; dilution 1:50; cat. no. MRQ-1; Beijing Zhongshan Jinqiao Biotechnology Co., Beijing, China), erythroblast transformation-specific-related gene (ERG; dilution 1:100; cat. no. EPR3864; Ventana Medical Systems, Tucson, AZ, USA), and cytokeratin (dilution 1:50; cat. no. M351501-2; Dako; Agilent Technologies, Inc.). Omission of primary antibody and substitution with non-specific immunoglobulins were used as negative controls. Appropriate positive controls were run concurrently for all antibodies tested. The slides were washed with PBS and incubated for $60 \mathrm{~min}$ at room temperature with a HRP-conjugated rabbit anti-mouse secondary antibody (dilution 1:200; cat. no. K0609; Agilent Technologies, Inc.). Following a further wash in PBS, the sections were stained with $0.05 \%$ diaminobenzidine containing $0.01 \%$ hydrogen peroxide. Finally, the sections were counterstained with hematoxylin, dehydrated and mounted. The immunohistochemical staining was observed with an optical microscope (BX43; Olympus Corp., Tokyo, Japan).

Statistical analysis. The overall survival (OS) was defined as the length of time between the date of diagnosis of angiosarcoma and date of mortality by any cause or the last follow-up date. The disease-free survival (DFS) was defined as the interval between the date of diagnosis and the date of recurrence, metastases or mortality. The significance of differences between groups was calculated using a paired Student's t-tests or $\chi^{2}$ tests. Fisher's exact tests were used to conducted categorical variables presented as counts and percentages. Univariate analyses were performed using the Kaplan-Meier method, and the survival difference between groups was assessed using the log-rank test. Cox proportional hazards regression model was used for multivariate survival analysis. $\mathrm{P}<0.05$ was considered to indicate a statistically significant difference. SPSS version 16.0 (SPSS, Inc., Chicago, IL, USA) was used to analyze all data.

\section{Results}

Clinical characteristics. The head and neck (64 cases) were the most common primary sites, particularly the scalp. A total of 22 tumors occurred in the bone, and the 22 tumors occurring in the trunk included post mediastinum (4 cases), chest wall ( 2 cases), shoulder back (6 cases), abdominal cavity ( 2 cases), buttock ( 3 cases) and inguinal region (5 cases). A total of 15 tumors developed in the extremities. The spleen (14 cases), heart (10 cases), lung (6 cases) and liver (4 cases) were the most commonly involved internal organs. In contrast, 33 tumors occurred in a superficial organ, the breast. Other uncommon organs included the ovary ( 2 cases), adrenal gland ( 2 cases), ileum (1 case), colon (1 case), thyroid gland (1 case), dura matter (1 case) and penis (1 case). The origin of the primary site was not defined in 1 case. According to the tumor location, 200 cases were divided into four groups: i) Tumors involving the head and neck; ii) tumors involving breast; iii) tumors involving viscera (including internal organs and bone); and iv) tumors involving soft tissue (including trunk and extremities).

In total, 97 males and 103 females were included in the present study. The mean and median age was 52.2 and 53 years (range, 4-91 years), respectively. Clinical characteristics are summarized in Table I. Male preponderance was observed in cases occurring in the head and neck (male/female ratio: 2.2:1). In contrast, all the patients except 1 with primary breast angiosarcoma were females. However, the patients with angiosarcoma of solid organs and soft tissue exhibited a similar distribution between sexes. As demonstrated in Table I, a significantly increasing mean age was observed in patients with tumor of breast, viscera, soft tissue and head $(\mathrm{P}<0.001)$. The peak incidence of angiosarcoma in the head and neck was in the sixth decade of life, while the peak age of incidences of breast angiosarcoma was younger compared with the peak ages in other sites.

The vast majority of head and neck angiosarcoma presented as bruise-like patches, violaceous nodules or plaques on the scalp in the present study. On progression, the lesions ulcerated and bled; patients complained of bleeding upon combing their hair. Satellite lesions were observed in certain patients. It has been reported that angiosarcoma of the head and neck in the elderly usually progresses slowly (6). In the present study, the interval between cutaneous lesion detection and tumor diagnosis was 1 month-40 years, with a median interval of 4.5 months. The most common symptom of angiosarcoma in soft tissue was an enlarged painful mass. Patients with primary 
Table I. Clinicopathological features of 200 patients with angiosarcoma.

\begin{tabular}{|c|c|c|c|c|c|}
\hline Clinicopathological features & $\begin{array}{c}\text { Total } \\
(\mathrm{n}=200)\end{array}$ & $\begin{array}{l}\text { Viscera } \\
(n=65)\end{array}$ & $\begin{array}{l}\text { Head and neck } \\
\qquad(n=64)\end{array}$ & $\begin{array}{l}\text { Soft tissue } \\
\quad(n=37)\end{array}$ & $\begin{array}{l}\text { Breast } \\
(n=33)\end{array}$ \\
\hline Male/female & $97 / 103$ & $32 / 33$ & $44 / 20$ & $20 / 17$ & $1 / 32$ \\
\hline Mean age, years & 52.3 & 45.5 & 66.0 & 52.6 & 38.6 \\
\hline$<65$ & 137 & 55 & 25 & 25 & 31 \\
\hline$\geq 65$ & 63 & 10 & 39 & 12 & 2 \\
\hline Mean size, $\mathrm{cm}$ & 5.2 & 6.4 & 4.5 & 4.6 & 5.1 \\
\hline$<5$ & 57 & 12 & 15 & 18 & 12 \\
\hline$\geq 5$ & 50 & 19 & 13 & 9 & 9 \\
\hline Unknown & 93 & 34 & 36 & 10 & 12 \\
\hline \multicolumn{6}{|l|}{ Tumor differentiation } \\
\hline High grade & 94 & 29 & 42 & 20 & 3 \\
\hline Low grade & 106 & 36 & 22 & 17 & 30 \\
\hline Epithelioid appearance & 37 & 14 & 10 & 13 & 0 \\
\hline \multicolumn{6}{|l|}{ Tumor recurrence/metastasis } \\
\hline Yes & 63 & 21 & 14 & 16 & 12 \\
\hline No & 50 & 15 & 15 & 9 & 11 \\
\hline Unknown & 87 & 29 & 35 & 12 & 20 \\
\hline \multicolumn{6}{|l|}{ Metastatic site } \\
\hline Bone & 18 & 11 & 3 & 3 & 1 \\
\hline Lung & 18 & 5 & 2 & 9 & 2 \\
\hline Liver & 8 & 6 & 2 & 0 & 0 \\
\hline Lymph node & 8 & 4 & 2 & 2 & 0 \\
\hline Trunk & 6 & 0 & 0 & 2 & 4 \\
\hline Parotid gland & 4 & 0 & 4 & 0 & 0 \\
\hline Breast & 3 & 0 & 0 & 0 & 3 \\
\hline Retroperitoneum & 2 & 2 & 0 & 0 & 0 \\
\hline Pleura & 1 & 1 & 0 & 0 & 0 \\
\hline Spleen & 1 & 1 & 0 & 0 & 0 \\
\hline \multicolumn{6}{|l|}{ Treatment modality } \\
\hline Multimodal therapy & 56 & 18 & 11 & 13 & 14 \\
\hline Surgery + radiotherapy & 18 & 5 & 5 & 4 & 4 \\
\hline Surgery + chemotherapy & 33 & 12 & 5 & 9 & 7 \\
\hline Surgery + radiotherapy + chemotherapy & 5 & 1 & 1 & 0 & 3 \\
\hline Non-multimodal therapy & 67 & 22 & 20 & 14 & 11 \\
\hline Surgery & 64 & 21 & 18 & 14 & 11 \\
\hline Radiotherapy & 2 & 1 & 1 & 0 & 0 \\
\hline Chemotherapy & 1 & 0 & 1 & 0 & 0 \\
\hline Unknown & 77 & 25 & 33 & 10 & 8 \\
\hline \multicolumn{6}{|l|}{ Survival, \% } \\
\hline 5-year disease-free survival & 19.3 & 19.0 & 0 & 18.7 & 34.8 \\
\hline 5-year overall survival & 40.8 & 37.0 & 28.0 & 36.6 & 87.5 \\
\hline
\end{tabular}

breast angiosarcoma usually presented with a palpable mass, with rapid growth resulting in prominent enlargement of the involved breast and a bluish discoloration of the overlying skin. Patients with cardiac angiosarcoma or pulmonary angiosarcoma presented with dyspnea, cough or hemoptysis. The clinical presentation of angiosarcoma of viscera was rather variable, including abdominal pain, hepatosplenomegaly, anemia and even life-threatening hemorrhage. Pain was the most common clinical symptom of primary angiosarcoma of the bone.

There were 2 cases of post-radiation angiosarcoma included in the present study. One patient had received radiotherapy for nasopharynx cancer. Angiosarcoma developed in the radiation fields 35 years following the radiotherapy. The other patient exhibited a previous history of malignant lymphoma in the tongue root, and received radiotherapy. A total of 20 years 

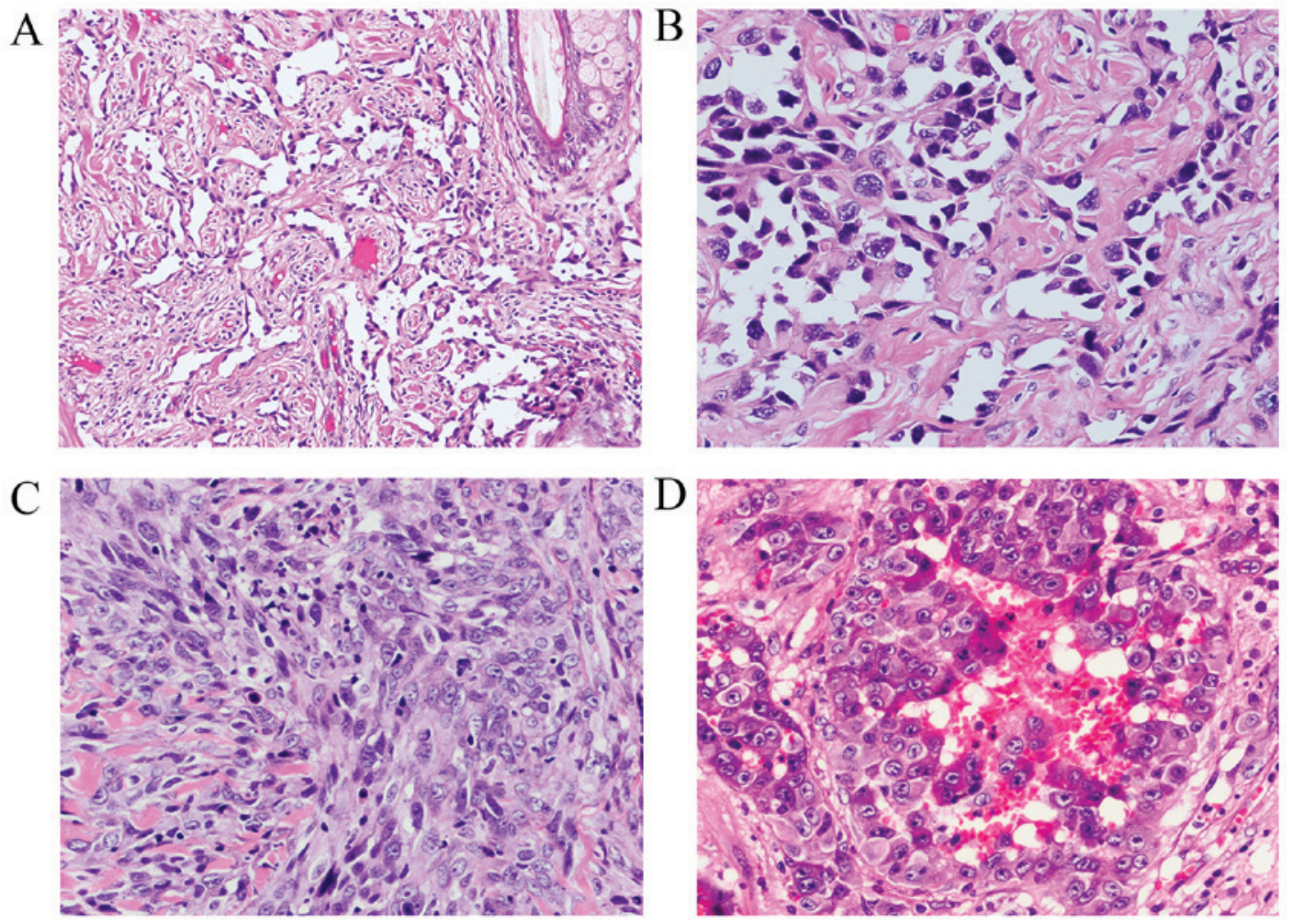

Figure 1. Representative microscopic images of the appearance of angiosarcomas. Low grade angiosarcoma demonstrated (A) irregular, anastomosing vascular channels (hematoxylin and eosin staining, original magnification, x200). (B) The ramifying channels were lined with atypical endothelial cells (hematoxylin and eosin staining, original magnification, x400). (C) High grade angiosarcoma exhibiting proliferation of high grade spindle tumor cells without clear vasoformation (hematoxylin and eosin staining, original magnification, $x 400$ ). (D) High grade angiosarcoma with epithelioid appearance with abundant amphophilic to lightly eosinophilic cytoplasm (hematoxylin and eosin staining, original magnification, x400).

following the radiotherapy, angiosarcoma was identified in the field of tongue root. A total of 3 cases of angiosarcoma of the extremities were associated with prolonged lymphedema (Stewart-Treves syndrome). A total of 2 patients developed upper limb angiosarcoma 10 and 14 years following radical mastectomy. The other patient with lower limb angiosarcoma exhibited a history of pain and swelling in the leg for 40 years. A history of 20-35 years of exposure to chemical products was noted in 6 patients. A total of 5 patients, including 2 patients with angiosarcoma involving the scalp, 2 patients with angiosarcoma involving the trunk and 1 patient with spleen angiosarcoma, exhibited a history of trauma. A total of 3 patients with breast angiosarcoma demonstrated a previous history of breast fibroadenoma, breast intraductal papilloma and thigh osteosarcoma, respectively. A total of 1 patient with a history of right breast human epidermal growth factor receptor 2-positive ductal adenocarcinoma was diagnosed with left breast angiosarcoma in the same year, and received modified radical mastectomy of the right breast and the extensive tumor resection of left breast, followed by Herceptin ${ }^{\circledR}$ therapy. One patient with pleural mesothelioma developed pulmonary angiosarcoma 9 years later.

Pathological features. Tumor size was recorded in 107 cases (mean, $5.2 \mathrm{~cm}$; median, $4.5 \mathrm{~cm}$; range, $0.5-15 \mathrm{~cm}$ ). According to the degree of vascular differentiation, cellular pleomorphism and mitotic activity, all cases were divided into low grade, and high grade (Table I). In low-grade cases, tumors contained irregular, complex anastomosing channels lined by atypical endothelial cells (Fig. 1A and B). In poorly differentiated areas, the high grade spindled tumor cells formed continuous sheets lacking vasoformation (Fig. 1C). In the present study, 37 cases $(18.5 \%, 37 / 200)$ exhibited an epithelioid appearance, and tumors were composed of epithelioid endothelial cells, which were classified as 'epithelioid angiosarcoma' and included in the high-grade group (Fig. 1D). However, the epithelioid appearance was not observed in the angiosarcoma of breast, spleen, liver and heart (Table I).

Immunohistochemistry is important in confirming the diagnosis of angiosarcoma, particularly for poorly differentiated cases. CD31 protein was detected in 105/110 tested cases $(95.5 \%)$ (Fig. 2A and B). CD34 staining was positive in 73/102 tested cases (71.6\%). ERG (Fig. 2C and D) and FLI1 (Fig. 2E and F), additional endothelial markers, demonstrated positive nuclear staining in all 20 tested cases. Cytokeratin was detected in 19/28 cases $(67.9 \%)$ of the epithelioid angiosarcomas examined.

Treatment. The therapeutic strategies of 123 patients were collected. According to the treatment modality, 123 patients were divided into two groups: i) 67 patients received non-multimodal treatment, including 64 patients treated with surgery alone, 2 patients treated with chemotherapy alone and 1 patient treated with radiotherapy alone; and ii) 56 patients received multidisciplinary treatment, including 33 patients treated with surgery and chemotherapy, 18 patients treated with surgery and radiotherapy, and 5 patients treated with a combination of surgery, radiotherapy and chemotherapy (Table I). 


\section{A}
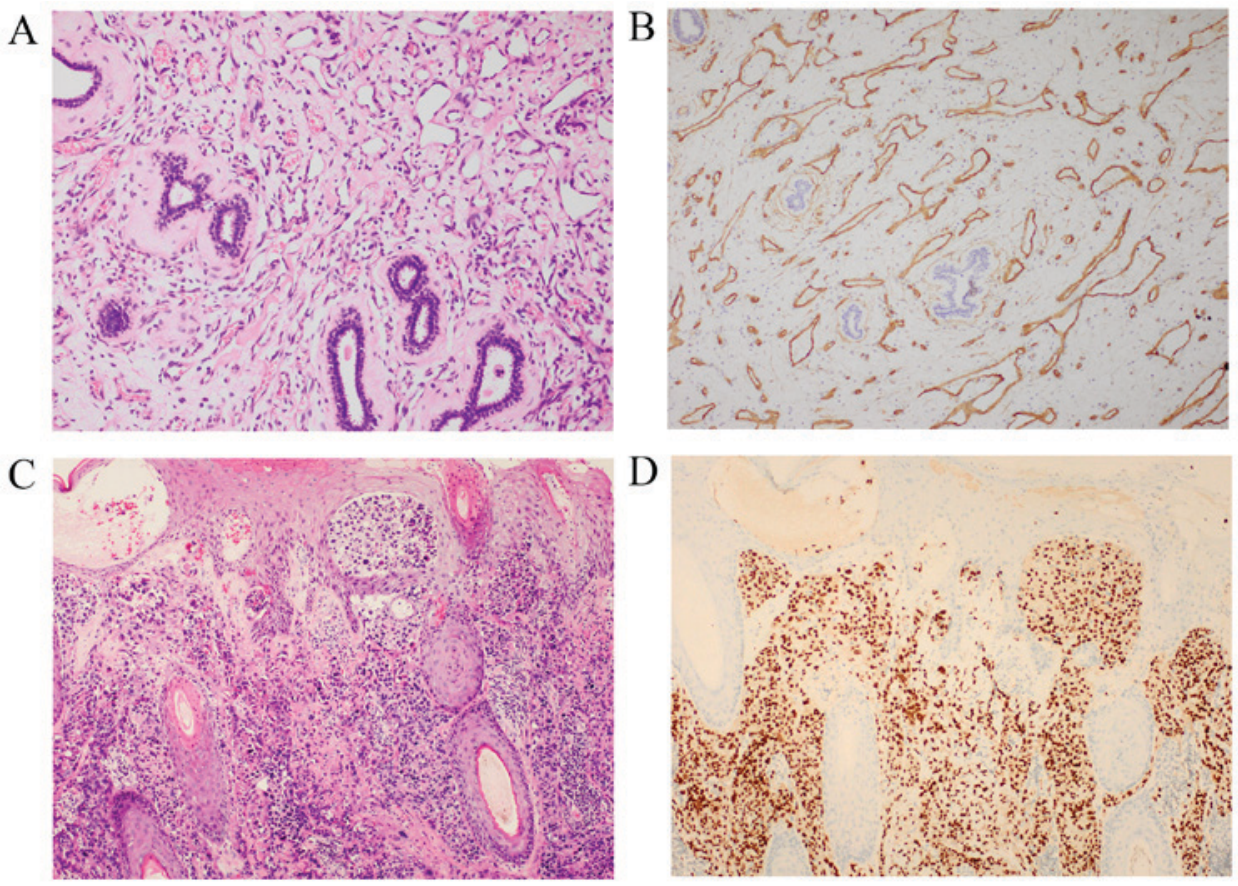

E
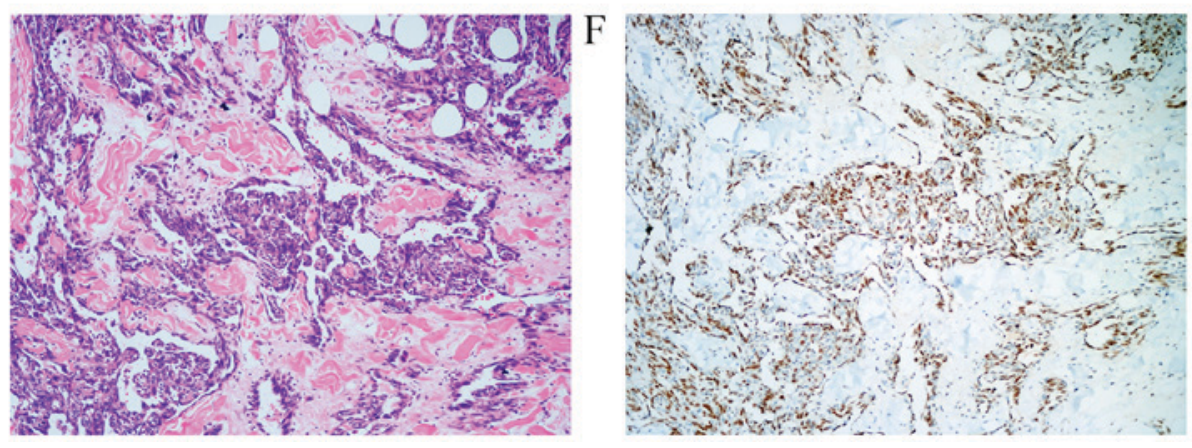

Figure 2. Immunohistochemical features of angiosarcoma. Tumor cells stained positively for the cluster of differentiation 31 antibody with (A) hematoxylin and eosin staining; and (B) immunohistochemical staining, original magnification, x100. Nuclear erythroblast transformation-specific-related gene staining with (C) hematoxylin and eosin staining; and (D) immunohistochemical staining, original magnification, x100, and Friend leukemia virus integration 1 staining with (E) hematoxylin and eosin staining; and (F) immunohistochemical staining, original magnification, $\mathrm{x} 200$ were positive in tumor cells.

Clinical follow-up. A total of 113 patients were followed up subsequent to angiosarcoma diagnosis until 31 March, 2014. The median follow-up duration was 13 months (range, 1-75 months). A total of 46 patients (40.7\%) succumbed to the disease at a median interval of 10 months (range, 1-70 months), 31 patients (27.4\%) lived with the disease at a median interval of 14 months (range, 1-61 months), and 34 patients (30.1\%) lived with no evidence of the disease at a median interval of 22.5 months (range, 3-75 months). A total of 2 patients (1.8\%) succumbed to hepatocellular carcinoma and lung adenocarcinoma. Tumor recurrence/metastasis was identified in 63 patients $(55.8 \%)$ at a median interval of 4 months (range, 0-40 months). At initial diagnosis, 18 (15.9\%) patients exhibited metastases. As summarized in Table I, bone (18 cases) and lung (18 cases) were the most common sites of tumor metastasis, followed by liver ( 8 cases), lymph nodes ( 8 cases), skin of the trunk (6 cases), parotid gland (4 cases), breast (3 cases), retroperitoneum ( 2 cases), pleura (1 case) and spleen (1 case). The parotid gland metastatic angiosarcomas originated exclusively from the head and neck, and the breast metastatic angiosarcomas originated exclusively from the contralateral breast.
In the present study, the median DFS and OS were 7, and 18 months, respectively. In all cases, the DFS at 1-, 3- and 5 -years were $38.1,22.1$, and $19.3 \%$, respectively. The OS at 1-, 3-, 5-years were 70.7, 50.0 and $40.8 \%$, respectively. DFS and OS at 5-year in patients with tumors involving the head and neck, breast, viscera, and soft tissue are summarized in Table I. There was no marked difference between viscera and soft tissue groups in DFS, and OS. Therefore, the two groups were combined together for the survival analyses. Tumor location had a significant effect on DFS ( $\mathrm{P}=0.032$; Fig. 3A) and OS ( $\mathrm{P}<0.001$; Fig. 3B). As demonstrated in Fig. 3, the patients with primary breast angiosarcoma exhibited a significantly improved DFS and OS compared with the patients in the other two groups, while the patients with involvement of angiosarcoma in head and neck exhibited relatively poor prognoses.

Prognostic factors of survival by univariate analysis are summarized in Table II. Tumor size $(\geq 5 \mathrm{~cm})$ and tumor differentiation exhibited an effect on DFS ( $\mathrm{P}=0.038$ and $<0.001$; Fig. $3 \mathrm{C}$ and $\mathrm{E}$, respectively) and $\mathrm{OS}(\mathrm{P}=0.008$ and $<0.001$; Fig. 3D and F, respectively). Tumor recurrence/metastasis was identified to be associated with DFS $(\mathrm{P}<0.001)$, but was not associated with OS $(\mathrm{P}=0.284)$. Age ( $\geq 65$ years) and treatment 

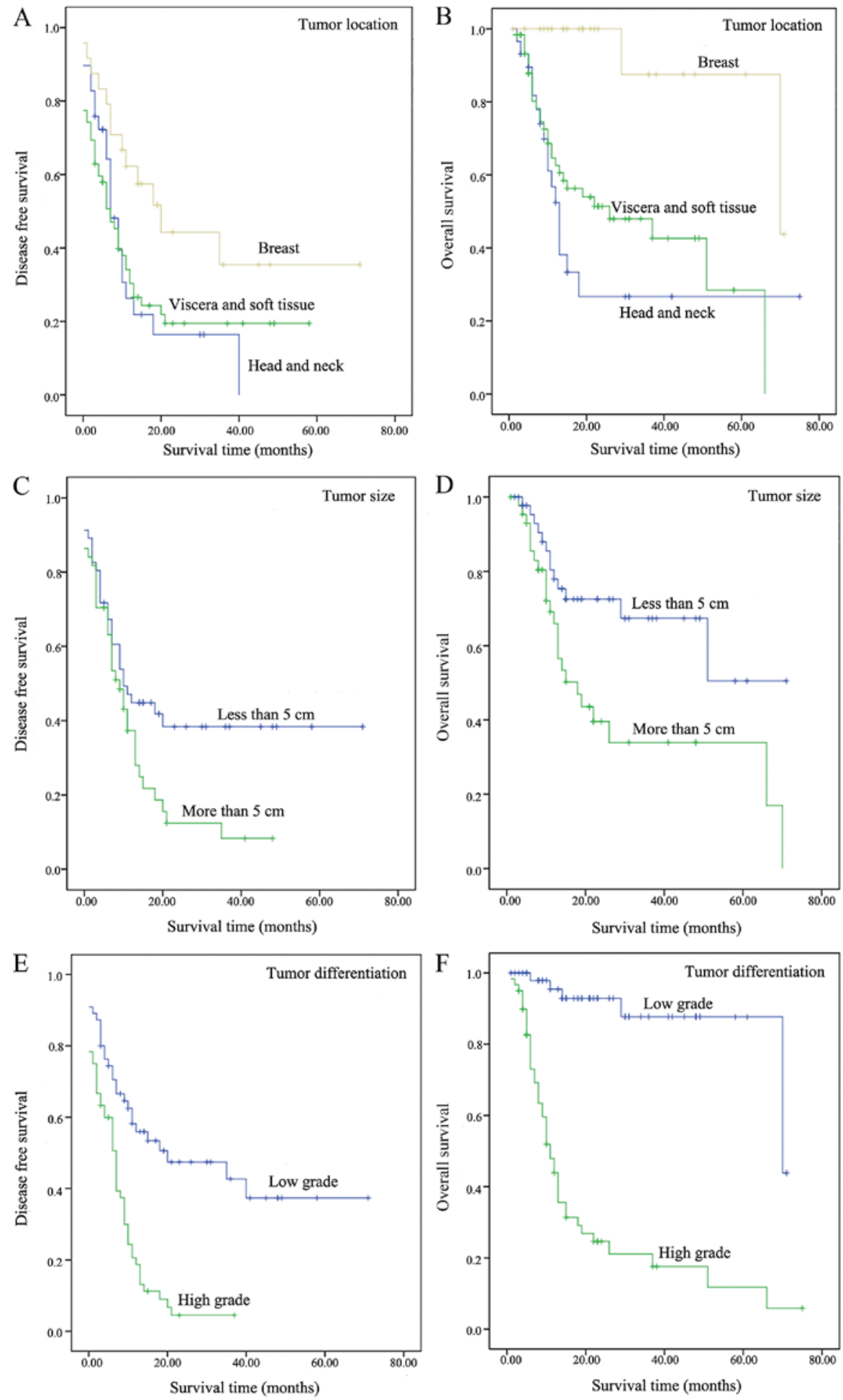

Figure 3. Kaplan-Meier curves comparing DFS and OS. (A) DFS and (B) OS of patients with different tumor location. (C) DFS and (D) OS of patients with different tumor sizes. (E) DFS and (F) OS of patients with different tumor differentiation. DFS, disease free survival; OS, overall survival.

modality exhibited a significant effect on $\mathrm{OS}(\mathrm{P}=0.003$ and $<0.001$, respectively), however, failed to predict DFS $(\mathrm{P}=0.172$ and 0.447 , respectively). Cox's analysis suggested that tumor recurrence/metastasis was an independent predictor of DFS [Hazard ratio (HR), 9.299; 95\% confidence interval (CI), 4.380-19.741; $\mathrm{P}<0.001]$ in patients with angiosarcoma, while tumor differentiation and treatment modality were the independent determinant of OS (HR, 8.439 and 2.672; 95\% CI, 2.615-27.229 and 1.057-6.756; $\mathrm{P}<0.001$ and 0.038, respectively).

\section{Discussion}

The aims of the present retrospective study were to describe the clinicopathological features and prognostic factors of 200 cases of angiosarcoma from a single Chinese medical institute. To the best of our knowledge, this series represents the largest study of this nature.

In accordance with previous studies, the head and neck $(32.0 \%, 64 / 200)$ were the most common primary sites for 
Table II. Univariate analysis of prognostic factors for survival.

\begin{tabular}{|c|c|c|c|c|}
\hline \multirow[b]{2}{*}{ Characteristic } & \multicolumn{2}{|c|}{ Disease-free survival rate } & \multicolumn{2}{|c|}{ Overall survival rate } \\
\hline & P-value & 5-year, $\%$ & P-value & 5 -year, \% \\
\hline Age, years & 0.172 & & 0.003 & \\
\hline$<65$ & & 21.4 & & 54.8 \\
\hline$\geq 65$ & & 0 & & 0 \\
\hline Tumor size, cm & 0.038 & & 0.008 & \\
\hline$<5$ & & 38.9 & & 51.7 \\
\hline$\geq 5$ & & 5.6 & & 31.6 \\
\hline Tumor differentiation & $<0.001$ & & $<0.001$ & \\
\hline High grade & & 0 & & 11.3 \\
\hline Low grade & & 36.9 & & 86.9 \\
\hline Tumor location & 0.032 & & $<0.001$ & \\
\hline Head and neck & & 0 & & 28.0 \\
\hline Viscera and soft tissue & & 18.9 & & 31.1 \\
\hline Breast & & 34.8 & & 87.5 \\
\hline Treatment modality & 0.447 & & $<0.001$ & \\
\hline Multimodal therapy & & 19.0 & & 53.5 \\
\hline Non-multimodal therapy & & 21.7 & & 31.7 \\
\hline Tumor recurrence/metastasis & $<0.001$ & & 0.284 & \\
\hline Yes & & 0 & & 31.0 \\
\hline No & & 60.4 & & 58.9 \\
\hline
\end{tabular}

angiosarcoma in the Chinese population, followed by the breast $(16.5 \%, 33 / 200)(3,4,7-9)$. Soft tissue angiosarcoma $(18.5 \%$, $37 / 200)$, which was composed of angiosarcoma involving the trunk $(11.0 \%, 22 / 200)$ and extremities $(7.5 \%, 15 / 200)$, also represented a large proportion of angiosarcoma. However, a relatively high proportion of pulmonary angiosarcoma $(3.0 \%$, $6 / 200$ ) was observed in this series. Lungs exhibit high frequencies of recurrence in patients with angiosarcoma $(9,10)$, and the incidence of primary pulmonary angiosarcoma was low; there are $<20$ cases identified in English language literature at present (11). In the present study, the diagnosis of a primary pulmonary angiosarcoma was based on excluding the presence of neoplasms located in any other sites by thorough clinical and radiological examination including computed tomography (CT), magnetic resonance imaging or positron emission tomography-CT.

The results revealed in the present study suggest that angiosarcoma is a family of sarcomas with distinctive behavior depending on the primary site. According to the primary site, all the cases in the present study were divided into four groups: i) Head and neck; ii) breast; iii) viscera (including internal organ and bone); and iv) soft tissue (including trunk and extremities). Overall, angiosarcomas exhibited a similar distribution between sexes (female/male, 103/97; ratio, 1.1:1). However, male predominance was only be observed in patients with head and neck angiosarcoma, and almost all angiosarcoma of the breast occurred exclusively in women. A similar distribution between sexes was observed in patients with angiosarcomas of the viscera and soft tissue. The peak incidence of angiosarcoma was also different among the four groups. Concomitant with previous studies $(12,13)$, angiosarcoma involving the head and neck were more common in the 60-80 age group, while breast angiosarcoma was observed commonly in the 30-50 age group. The incidence of angiosarcoma involving viscera and soft tissue in the patient population of the present study was only observed in the 40-60 age group, which was younger compared with previous studies in which those aged 60-70 experienced the peak incidence rate $(14,15)$.

The results of the present study also demonstrated that the primary sites of the tumor affect the prognosis, which may be used to classify angiosarcoma. DFS and OS were affected by the primary site of angiosarcoma in the present study. The head and neck angiosarcomas were associated with the poorest survival, with a 5-year OS of $28.0 \%$. However, the outcomes of patients with breast angiosarcomas in the present study were favorable, with a 5-year OS of $87.5 \%$. Such a favorable outcome was not consistent with the other studies investigating breast angiosarcoma $(16,17)$. Angiosarcomas of the breast may arise do novo, and occur as secondary tumors following radiation $(18,19)$. The two types of breast angiosarcoma may be biologically different. Secondary breast angiosarcoma exhibits a poorer prognosis compared with the primary angiosarcoma (20). The prognosis of primary angiosarcoma is favorable, particularly with low-grade differentiation (21). In the present study, all of the breast angiosarcomas were primary and 30/33 tumors were low-grade, which may explain the favorable biological behavior of these tumors. Soft tissue angiosarcoma is rare, and little is known regarding its clinicopathological features and prognosis. The outcome of patients with soft tissue angiosarcoma lacks consensus in the literature. Meis-Kindblom and Kindblom (14) 
suggested that angiosarcoma of soft tissue is a high-grade sarcoma with high rate of mortality (53\% of patients succumbed to the disease at a median interval of 11 months), while Fayette et al (3) demonstrated that the clinical outcome of soft tissue angiosarcoma was favorable (5-year survival rate, 74\%), and visceral angiosarcoma was more aggressive and suggested poorer prognosis. However, in the present study, the 5-year OS rates of angiosarcoma, involving solid organs and soft tissue similar were 37.0 and $36.6 \%$, respectively. Therefore, the clinicopathological features and prognosis of soft tissue, and viscera angiosarcoma require additional investigation.

Tumor size and tumor differentiation are important prognostic factors in soft tissue sarcoma (22), and their value in angiosarcoma may also be confirmed in the present study. The present univariate analysis confirmed that tumor size and histological grade are prognostic factors for DFS and OS. Tumors size $\geq 5 \mathrm{~cm}$ and high-grade histological differentiation predicted poor prognosis, as previously described $(7,8,10,23,24)$. Other factors predicted poor clinical outcome, including old age ( $\geq 65$ years) and tumor recurrence/metastasis. However, which prognostic factors were the most important was unclear. The present study identified that tumor differentiation and recurrence/metastasis were independent prognostic factors using multivariate survival analysis. Although certain parameters may not be independent prognostic factors, the present study hypothesizes that all the aforementioned factors mentioned should be considered by physicians when assessing the biological behavior of the tumor and choosing the therapeutic strategies.

At present, the treatment options for patients with angiosarcoma are controversial. The primary treatment of angiosarcoma was radical surgery with complete (R0) resection. Karpeh et al (25) suggested that the survival rate of patients with malignant of vascular tumors depends on R0 surgical resection. However, because of the invasive and multifocal nature of angiosarcoma, wide margins are difficult to achieve. Naka et al (26) indicated that multimodal treatment (surgery followed by chemotherapy and/or radiotherapy) was associated with improved survival. In the present study, patients were divided into two groups according to the mode of treatment: i) Non-multimodal treatment including surgery alone, which was the primary mode of simple treatment, chemotherapy alone and radiotherapy alone; and ii) multimodal treatment, including surgery followed by chemotherapy or radiotherapy, and a combination of surgery, chemotherapy and radiotherapy. The present multivariate analysis revealed that the multimodal treatment was an independent favorable prognostic factor in angiosarcoma.

The majority of angiosarcoma arises spontaneously, but several risk factors are associated with the development of angiosarcoma: Lymphoedema (27), radiation (28), various chemicals $(29,30)$ and immunodeficiency $(31)$. In the present study, there were 2 cases of angiosarcoma arising at the radiation fields, which were considered radiation-associated angiosarcoma. Breast angiosarcoma, which was associated with radiotherapy for an epithelial breast cancer (secondary), comprised the majority of radiation-associated angiosarcoma (32). However, the 2 patients with radiation-associated angiosarcoma in the present study exhibited previous histories of nasopharynx cancer and malignant lymphoma, respectively, and all the cases of breast angiosarcoma were primary.
Chronic lymphedema is an additional well-defined cause of angiosarcoma, which is known as Stewart-Treves syndrome. A total of $0.6-8.0 \%$ of all angiosarcoma have occurred under such conditions $(3,33)$. In the present study, 3 cases of angiosarcoma $(1.5 \%)$ were associated with prolonged lymphedema. A total of 2 patients exhibited upper limb angiosarcoma arising from chronic lymphedema 10 and 14 years following radical mastectomy. The other patient with lower limb angiosarcoma exhibited a history of limb edema for 40 years.

In summary, angiosarcomas are a family of sarcomas with distinct clinical behavior depending on the primary sites, which effects the clinical outcome. Tumor recurrence/metastasis, tumor differentiation and treatment modality are the independent factors for prognosis. In the present study, multimodal treatment may improve the clinical outcome of patients with angiosarcoma.

\section{Acknowledgements}

The abstract was selected for a poster presentation at the United States and Canadian Academy of Pathology's 104th Annual Meeting, March 21-27, 2015 in Boston, MA and published as abstract no. 100 in Lab. Invest. Vol. 95 (Suppl 1) p28A: 2015. This study was financially supported by Shanghai Key Developing Disciplines (grant no. 2015ZB0201) and Shanghai Hospital Development Center Emerging Advanced Technology Joint Research Project (grant no. HDC12014105).

\section{References}

1. Rouhani P, Fletcher CD, Devesa SS and Toro JR: Cutaneous soft tissue sarcoma incidence patterns in the U.S.: An analysis of 12,114 cases. Cancer 113: 616-627, 2008.

2. Toro JR, Travis LB, Wu HJ, Zhu K, Fletcher CD and Devesa SS: Incidence patterns of soft tissue sarcomas, regardless of primary site, in the surveillance, epidemiology and end results program, 1978-2001: An analysis of 26,758 cases. Int J Cancer 119: 2922-2930, 2006.

3. Fayette J, Martin E, Piperno-Neumann S, Le Cesne A, Robert C, Bonvalot $\mathrm{S}$, Ranchère $\mathrm{D}$, Pouillart $\mathrm{P}$, Coindre JM and Blay JY: Angiosarcomas, a heterogeneous group of sarcomas with specific behavior depending on primary site: a retrospective study of 161 cases. Ann Oncol 18: 2030-2036, 2007.

4. Fury MG, Antonescu CR, Van Zee KJ, Brennan MF and Maki RG: A 14-year retrospective review of angiosarcoma: Clinical characteristics, prognostic factors, and treatment outcomes with surgery and chemotherapy. Cancer J 11: 241-247, 2005.

5. Fletcher CDM, Bridge JA, Hogendoorn PCW and Mertens F (eds): WHO Classification of Tumours of Soft Tissue and Bone. 4th edition. IARC Press, Lyon, pp56, 2013.

6. Farid M, Ong WS, Lee MJ, Jeevan R, Ho ZC, Sairi AN, Soh LT, Poon D, Teh J, Chin F, et al: Cutaneous versus non-cutaneous angiosarcoma: Clinicopathologic features and treatment outcomes in 60 patients at a single Asian cancer centre. Oncology 85: 182-190, 2013.

7. Mark RJ, Poen JC, Tran LM, Fu YS and Juillard GF: Angiosarcoma. A report of 67 patients and a review of the literature. Cancer 77: 2400-2406, 1996.

8. Abraham JA, Hornicek FJ, Kaufman AM, Harmon DC, Springfield DS, Raskin KA, Mankin HJ, Kirsch DG, Rosenberg AE, Nielsen GP, et al: Treatment and outcome of 82 patients with angiosarcoma. Ann Surg Oncol 14: 1953-1967, 2007.

9. Naka N, Ohsawa M, Tomita Y, Kanno H, Uchida A and Aozasa K: Angiosarcoma in Japan. A review of 99 cases. Cancer 75: 989-996, 1995.

10. Lahat G, Dhuka AR, Lahat S, Smith KD, Pollock RE, Hunt KK, Ravi V, Lazar AJ and Lev D: Outcome of locally recurrent and metastatic angiosarcoma. Ann Surg Oncol 16: 2502-2509, 2009. 
11. Kakegawa S, Kawashima O, Ibe T, Ujita M, Iwashina M, Nakano T and Shimizu K: A case of primary angiosarcoma of the lung presenting as a hemorrhagic bronchial tumor. Ann Thorac Cardiovasc Surg 18: 347-351, 2012.

12. Letsa I, Benson C, Al-Muderis O and Judson I: Angiosarcoma of the face and scalp: Effective systemic treatment in the older patient. J Geriatr Oncol 5: 276-280, 2014.

13. Bae SY, Choi MY, Cho DH, Lee JE, Nam SJ and Yang JH: Large clinical experience of primary angiosarcoma of the breast in a single Korean medical institute. World J Surg 35: 2417-2421, 2011

14. Meis-Kindblom JM and Kindblom LG: Angiosarcoma of soft tissue: A study of 80 cases. Am J Surg Pathol 22: 683-697, 1998.

15. Zheng YW, Zhang XW, Zhang JL, Hui ZZ, Du WJ, Li RM and Ren XB: Primary hepatic angiosarcoma and potential treatment options. J Gastroenterol Hepatol 29: 906-911, 2014.

16. Vorburger SA, Xing Y, Hunt KK, Lakin GE, Benjamin RS, Feig BW, Pisters PW, Ballo MT, Chen L, Trent J III, et al: Angiosarcoma of the breast. Cancer 104: 2682-2688, 2005.

17. Hodgson NC, Bowen-Wells C, Moffat F, Franceschi D and Avisar E: Angiosarcomas of the breast: A review of 70 cases. Am J Clin Oncol 30: 570-573, 2007.

18. Biswas T, Tang P, Muhs A and Ling M: Angiosarcoma of the breast: A rare clinicopathological entity. Am J Clin Oncol 32: 582-586, 2009

19. Monroe AT, Feigenberg SJ and Mendenhall NP: Angiosarcoma after breast-conserving therapy. Cancer 97: 1832-1840, 2003

20. Fraga-Guedes C, Gobbi H, Mastropasqua MG, Botteri E, Luini A and Viale G: Primary and secondary angiosarcomas of the breast: A single institution experience. Breast Cancer Res Treat 132: 1081-1088, 2012

21. Rosen PP, Kimmel M and Ernsberger D: Mammary angiosarcoma. The prognostic significance of tumor differentiation. Cancer 62: 2145-2151, 1988.

22. Van Glabbeke M, van Oosterom AT, Oosterhuis JW, Mouridsen H, Crowther D, Somers R, Verweij J, Santoro A, Buesa J and Tursz T: Prognostic factors for the outcome of chemotherapy in advanced soft tissue sarcoma: An analysis of 2,185 patients treated with anthracycline-containing first-line regimens-a European Organization for Research and Treatment of Cancer Soft Tissue and Bone Sarcoma Group Study. J Clin Oncol 17: 150-157, 1999.
23. Pawlik TM, Paulino AF, McGinn CJ, Baker LH, Cohen DS, Morris JS, Rees R and Sondak VK: Cutaneous angiosarcoma of the scalp: A multidisciplinary approach. Cancer 98: 1716-1726, 2003.

24. Sher T, Hennessy BT, Valero V, Broglio K, Woodward WA, Trent J, Hunt KK, Hortobagyi GN and Gonzalez-Angulo AM: Primary angiosarcomas of the breast. Cancer 110: 173-178, 2007.

25. Karpeh MS Jr, Caldwell C, Gaynor JJ, Hajdu SI and Brennan MF: Vascular soft-tissue sarcomas. An analysis of tumor-related mortality. Arch Surg 126: 1474-1481, 1991.

26. Naka N, Ohsawa M, Tomita Y, Kanno H, Uchida A, Myoui A and Aozasa K: Prognostic factors in angiosarcoma: A multivariate analysis of 55 cases. J Surg Oncol 61: 170-176, 1996.

27. Stewart FW and Treves N: Lymphangiosarcoma in postmastectomy lymphedema; a report of six cases in elephantiasis chirurgica. Cancer 1: 64-81, 1948.

28. Huang J and Mackillop WJ: Increased risk of soft tissue sarcoma after radiotherapy in women with breast carcinoma. Cancer 92: 172-180, 2001.

29. Bosetti C, La Vecchia C, Lipworth L and McLaughlin JK: Occupational exposure to vinyl chloride and cancer risk: A review of the epidemiologic literature. Eur J Cancer Prev 12: 427-430, 2003

30. Ron E: Cancer risks from medical radiation. Health Phys 85: 47-59, 2003.

31. Ahmed I and Hamacher KL: Angiosarcoma in a chronically immunosuppressed renal transplant recipient: Report of a case and review of the literature. Am J Dermatopathol 24: 330-335, 2002.

32. Lucas DR: Angiosarcoma, radiation-associated angiosarcoma, and atypical vascular lesion. Arch Pathol Lab Med 133: 1804-1809, 2009

33. Perez MC, Padhya TA, Messina JL, Jackson RS, Gonzalez RJ, Bui MM, Letson GD, Cruse CW, Lavey RS, Cheong D, et al: Cutaneous angiosarcoma: A single-institution experience. Ann Surg Oncol 20: 3391-3397, 2013. 